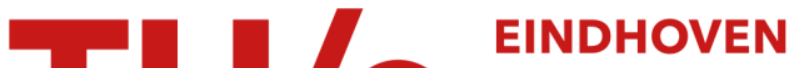 UNIVERSITY OF TECHNOLOGY
}

\section{Relaxation of a strained quantum well at a cleaved surface. Part II: effect of cubic symmetry}

\section{Citation for published version (APA):}

Davies, J. H., Offermans, P., \& Koenraad, P. M. (2005). Relaxation of a strained quantum well at a cleaved surface. Part II: effect of cubic symmetry. Journal of Applied Physics, 98(5), 053504-1/10. [053504]. https://doi.org/10.1063/1.2030415

DOI:

$10.1063 / 1.2030415$

Document status and date:

Published: 01/01/2005

\section{Document Version:}

Publisher's PDF, also known as Version of Record (includes final page, issue and volume numbers)

\section{Please check the document version of this publication:}

- A submitted manuscript is the version of the article upon submission and before peer-review. There can be important differences between the submitted version and the official published version of record. People interested in the research are advised to contact the author for the final version of the publication, or visit the $\mathrm{DOI}$ to the publisher's website.

- The final author version and the galley proof are versions of the publication after peer review.

- The final published version features the final layout of the paper including the volume, issue and page numbers.

Link to publication

\section{General rights}

Copyright and moral rights for the publications made accessible in the public portal are retained by the authors and/or other copyright owners and it is a condition of accessing publications that users recognise and abide by the legal requirements associated with these rights.

- Users may download and print one copy of any publication from the public portal for the purpose of private study or research.

- You may not further distribute the material or use it for any profit-making activity or commercial gain

- You may freely distribute the URL identifying the publication in the public portal.

If the publication is distributed under the terms of Article 25fa of the Dutch Copyright Act, indicated by the "Taverne" license above, please follow below link for the End User Agreement:

www.tue.nl/taverne

Take down policy

If you believe that this document breaches copyright please contact us at:

openaccess@tue.nl

providing details and we will investigate your claim. 


\title{
Relaxation of a strained quantum well at a cleaved surface. Part II: Effect of cubic symmetry
}

\author{
John H. Davies ${ }^{\text {a) }}$ \\ Department of Electronics and Electrical Engineering, Glasgow University, Glasgow, G12 8QQ, \\ United Kingdom \\ Peter Offermans and Paul M. Koenraad \\ Inter-University Research Institute on Communication Technology (COBRA), Department of Physics, \\ Eindhoven University of Technology, P.O. Box 513, NL-5600MB Eindhoven, The Netherlands
}

(Received 4 February 2005; accepted 11 July 2005; published online 2 September 2005)

In a previous paper [J. H. Davies, D. M. Bruls, J. W. A. M. Vugs, and P. M. Koenraad, J. Appl. Phys. 91, 4171 (2002). Part I.] we compared theory and experiment for the relaxation at a cleaved surface of a strained quantum well of InGaAs in GaAs. The measurements were taken with a scanning tunneling microscope and the analytic calculation used classical elastic theory for a linear, isotropic, homogeneous medium. Qualitative agreement was good but the theory gave only about $80 \%$ of the observed displacement. We have therefore extended the calculation to explore the effect of cubic symmetry and the orientation of the cleaved surface. The "strain suppression" method reduces the problem to the response of a half space to traction on its surface. We have calculated this for orthotropic symmetry, which includes the common orientations of orthorhombic, tetragonal, hexagonal, and cubic crystals. Anisotropy has no effect on the shape of the relaxed surface but the magnitude of relaxation changes. For cubic material there is no effect on the strain along the direction of growth if the cleaved surface is a $\{001\}$ plane and a reduction of a few percent for a $\{011\}$ plane, which is the case of experimental interest. The outward relaxation is reduced by about $20 \%$ due to cubic symmetry for a $\{001\}$ plane because the shear stiffness of GaAs is higher than in the isotropic model, and is a further $10 \%$ smaller for a $\{011\}$ plane. Thus the results for cubic symmetry lie further from the measurements than those calculated for isotropic material. Interfacial forces may contribute to this discrepancy but we suggest that nonlinear elasticity is probably responsible. (c) 2005 American Institute of Physics. [DOI: 10.1063/1.2030415]

\section{INTRODUCTION}

The use of heterostructures that contain coherently strained layers of materials with different lattice constants is growing rapidly. It is particularly important to characterize them accurately because errors in composition affect the functional properties both directly and through the built-in strain. Both the composition and strain can be measured by cleaving the sample and scanning the exposed cross section with a probe. The composition can be found immediately by counting atoms and the strain can be measured in two ways.

- Strain in the plane of the surface can be deduced from the spacing between atoms. This needs a highresolution cross-sectional scanning tunneling microscope $(\mathrm{X}-\mathrm{STM}){ }^{1,2}$

- Distortion also occurs normal to the surface and can be measured without the need for atomic resolution in the plane: atomic force microscopy (AFM) may be sufficient. ${ }^{3-6}$

The composition can be deduced from the natural lattice spacing of the material. However, the region near the surface relaxes inhomogeneously to relieve its elastic energy so the spacing between atoms on the surface differs from the lattice

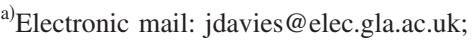

URL: www.elec.gla.ac.uk/jdavies
}

constant in the bulk. A calculation of the relaxation is therefore required. Numerical methods ${ }^{3-5}$ must be used for a full solution but the elastic field in a cleaved sample that contains a single, uniform, strained layer or a superlattice can be found analytically ${ }^{6-11}$ subject to certain simplifications.

In a previous paper, ${ }^{6}$ to be cited as I, we compared measurements and calculations for a strained quantum well with uniform composition of $\mathrm{In}_{x} \mathrm{Ga}_{1-x}$ As surrounded by GaAs. The cleaved surface is sketched in Fig. 1(a) with its relaxation shown in Fig. 1(b). The apparent lattice constant, defined as the separation between rows of atoms on the surface, and the distortion normal to the cleaved surface were measured along the direction of growth. Atoms of In could be distinguished from those of $\mathrm{Ga}$ on the surface and were counted to determine the composition. This was confirmed by photoluminescence and x-ray diffraction.
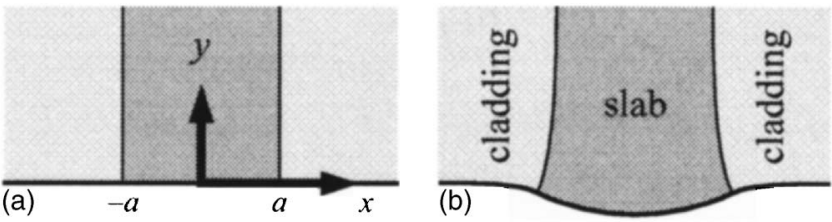

FIG. 1. (a) Cladding (GaAs) around a slab (quantum well of $\operatorname{In}_{x} \mathrm{Ga}_{1-x} \mathrm{As}$, with a larger lattice constant), after a surface has been cleaved but before it has relaxed. The sample occupies the half space $y>0$ with $x$ along the direction of growth. (b) Sketch of sample after it has relaxed. 
The calculation was based on the "strain suppression" approach to thermoelasticity, ${ }^{12,13}$ which reduces it to a boundary-value problem. This was solved using standard results from contact mechanics for a semi-infinite region. ${ }^{14}$ It was assumed that the medium was linear, homogeneous, and isotropic with Poisson's ratio $\nu$. Take $x$ as the axis of growth and $y$ as the inward normal to the cleaved surface, as shown in Fig. 1. The sample is assumed to be of infinite extent along $z$, in which case relaxation takes place under conditions of plane strain. This choice of axes is regrettably different from I but is more consistent with the usage for twodimensional systems. The slab or quantum well lies in $|x|$ $<a$ and its natural, linear dimensions, given by its lattice constant, exceed those of its surroundings (the cladding) by a fraction $\varepsilon_{0}$. The principal results for the surface were as follows.

(1) The lattice constant of the cladding is unaffected despite its distortion when the slab relaxes.

(2) The lattice constant of the slab increases uniformly along the direction of growth $x$ by a fraction $\varepsilon_{x x}^{(\text {tot })}$ $=(1+2 \nu) \varepsilon_{0}$.

(3) There is an outward relaxation of the surface given by

$$
\begin{aligned}
-u_{y}(x, y=0)= & \frac{A_{0} \varepsilon_{0}}{\pi}\left[(x-a) \ln \left|\frac{x-a}{a}\right|\right. \\
& \left.-(x+a) \ln \left|\frac{x+a}{a}\right|\right],
\end{aligned}
$$

with $A_{0}=2(1+\nu)$.

For an arbitrary variation of composition along $x$, the strain on the surface is proportional to the local composition and the slope is given by a Hilbert transform of the composition.

Qualitative agreement between the calculation and experiment was excellent. There was no detectable change in the lattice constant of the cladding. Within the slab the lattice constant was uniformly increased over its natural value. The shape of the surface was also given well by Eq. (1.1). However, the degree of relaxation predicted by the calculation was only about $80 \%$ of that observed. Several reasons for this discrepancy were considered in I. There are possible difficulties with the measurement of $u_{y}$ but these do not affect $\varepsilon_{x x}$, which has a built-in control because the lattice constant of the cladding matched that of GaAs. Deficiencies in the theory include the assumptions of isotropy, homogeneity, and linearity. There may also be forces associated with the interfaces and surface, and piezoelectricity was neglected.

Previous calculations have shown that cubic symmetry has an appreciable effect on relaxation ${ }^{5,15,16}$ and we have therefore extended our calculation to include this. We find that the shape of the surface remains the same but the magnitude of relaxation changes. Unfortunately the results lie slightly further away from the measurements than the previous calculation (as predicted in I) so this does not explain why the theoretical result for the relaxation is too small.

The effective symmetry of a two-dimensional problem depends on both the intrinsic symmetry of the material and on the orientation of the sample. Some previous analytic calculations for the relaxation of strained layers ${ }^{8,9}$ treated cubic symmetry for systems where the natural axes of the problem (xyz in Fig. 1) coincided with the cubic axes of the crystal. We shall call this the "cubic aligned" orientation. Unfortunately the cubic semiconductors cleave on $\{011\}$ planes, which lowers the effective symmetry. The direction of growth, $x$, lies along the cubic axis [100] but $y$ points along [011] or [01 $\overline{1}]$, not [010]. Thus the elastic properties do not show explicit cubic symmetry when expressed using the $x y z$ axes in this "cubic rotated" orientation. The relaxation of a superlattice has been calculated for both orientations ${ }^{11}$ using a Fourier series. This is a little cumbersome for a single strained layer, compared with the local results for the strain at the surface that we found in I, and we have therefore performed a direct calculation in real space. We also permit lower symmetry and assume only that there is a mirror plane normal to each of the $x y z$ axes, giving orthotropic symmetry. This includes the cleaved, cubic semiconductors. Conveniently it also embraces hexagonal crystals, such as the nitride semiconductors, whose elastic properties have transverse isotropy in the $y z$ plane if growth is along $x$.

We have again neglected piezoelectricity. This has an insignificant effect on the elastic response of GaAs but the omission is questionable for the nitrides. ${ }^{17}$ Solutions are available for both two- ${ }^{18}$ and three-dimensional ${ }^{19,20}$ inclusions in a piezoelectric half space but are considerably more complicated than the theory presented here.

\section{APPROACH}

The calculation follows the same strategy as in I except for the choice of axes. It is assumed that the system of slab and cladding has the same elastic properties everywhere and that the response is linear, but the assumption of isotropic behavior is relaxed. There are nine independent elastic constants in an orthotropic material, although not all are needed in this calculation. Normal stresses and strains are related by three equations of the form

$$
\sigma_{x x}=c_{11} \varepsilon_{x x}+c_{12} \varepsilon_{y y}+c_{13} \varepsilon_{z z},
$$

where the coefficients are independent apart from the symmetry $c_{i j}=c_{j i}$. Shear stresses and strains are related by $\sigma_{x y}$ $=2 c_{66} \varepsilon_{x y}$ and similar expressions, where the coefficients are again independent. The mismatch between the slab and cladding is assumed to be the same in all three dimensions, defined by

$$
\varepsilon_{0}=\frac{a_{\text {slab }}-a_{\text {cladding }}}{a_{\text {cladding }}} .
$$

Here $a$ is the lattice constant, which is taken to be larger in the slab for the purpose of description. It would be trivial to treat a different mismatch for each dimension, as would be needed for hexagonal crystals. Most of the strain is in the slab and it might therefore be more appropriate to take $a_{\text {slab }}$ as the reference in the denominator, but the difference is insignificant within linear elastic theory.

The calculation follows the strain suppression approach to thermoelasticity as in I. Start with the slab and cladding separate, free of stress, and oriented normal to $x$. Next, compress the slab along $y$ and $z$ to reduce its lattice constant to 
that of the cladding, which requires strains $\varepsilon_{y y}^{(\text {slab })}=\varepsilon_{z z}^{\text {(slab) }}$ $=-\varepsilon_{0}$. The condition $\sigma_{x x}^{\text {(slab) }}=0$ along the direction of growth leads to

$$
\varepsilon_{x x}^{(\text {slab })}=\frac{c_{12}+c_{13}}{c_{11}} \varepsilon_{0}, \quad \varepsilon^{(\text {slab })}=-\frac{2 c_{11}-\left(c_{12}+c_{13}\right)}{c_{11}} \varepsilon_{0}
$$

for the extension of the slab along the direction of growth and the dilation. The stress required is

$$
\sigma_{y y}^{(\text {slab })}=-P_{0}=-\frac{c_{11}\left(c_{22}+c_{23}\right)-c_{12}\left(c_{12}+c_{13}\right)}{c_{11}} \varepsilon_{0},
$$

with a similar expression for $\sigma_{z z}^{(\text {slab) }}$. The slab can now be joined to the cladding without further distortion. Finally, the fictitious stress used to assemble the system must be canceled by applying the opposite stress to the edges of the slab exposed on the surfaces normal to $y$ and $z$ and allowing the material to relax. We shall assume that the sample is so large that each edge can be treated independently and study the single edge exposed on the plane $y=0$, shown in Fig. 1, treated as a semi-infinite region. Relaxation takes place as plane strain in $x$ and $y$ because of the infinite extent along $z$. This approach would not be appropriate for transmission electron microscopy, where the samples are thinned and a theory that treats two free surfaces is needed. ${ }^{7-11,21}$ In contrast, our sample for scanning probe microscopy is over $100 \mu \mathrm{m}$ thick along $z$ so this problem does not arise. Instead, the limiting factor is the thickness of the capping layer under which the quantum well is buried. This is very thin in many wafers but is $2 \mu \mathrm{m}$ thick in our sample, much larger than the region where significant relaxation occurs (about $20 \mathrm{~nm}$ ).

The relaxation is therefore given by the response of the half space $y>0$ to an applied stress $\sigma_{y y}(x, 0)=p(x)$, where $p(x)=P_{0}$ on the exposed edge of the slab $(|x|<a)$ and vanishes elsewhere. A general solution of this boundary-value problem will be given in Sec. III.

\section{SOLUTION OF BOUNDARY-VALUE PROBLEM}

The central problem is to find the elastic response of an orthotropic half space $y>0$ to a distribution of normal traction $\sigma_{y y}(x, 0)=p(x)$ on its free surface. For generality we will also include shear traction $\sigma_{x y}(x, 0)=q(x)$ and will use complex variables because general results are displayed more directly than with Fourier methods. ${ }^{7-10}$ The approach is taken from Green and Zerna, ${ }^{22}$ particularly Sec. 9.5; the Lekhnitskii or Stroh formalisms ${ }^{23}$ could be used instead.

\section{A. Airy function}

The stress is derived in the usual way from an Airy function $\chi$

$$
\sigma_{x x}=\frac{\partial^{2} \chi}{\partial y^{2}}, \quad \sigma_{x y}=-\frac{\partial^{2} \chi}{\partial x \partial y}, \quad \sigma_{y y}=\frac{\partial^{2} \chi}{\partial x^{2}} .
$$

This ensures that the stress satisfies the condition for equilibrium. The strain must also obey the single compatibility condition

$$
\frac{\partial^{2} \varepsilon_{x x}}{\partial y^{2}}-2 \frac{\partial^{2} \varepsilon_{x y}}{\partial x \partial y}+\frac{\partial^{2} \varepsilon_{y y}}{\partial x^{2}}=0 .
$$

The stresses for plane strain are given by

$$
\begin{aligned}
& \sigma_{x x}=c_{11} \varepsilon_{x x}+c_{12} \varepsilon_{y y}, \\
& \sigma_{y y}=c_{12} \varepsilon_{x x}+c_{22} \varepsilon_{y y}, \\
& \sigma_{x y}=2 c_{66} \varepsilon_{x y} .
\end{aligned}
$$

There is a further equation for $\sigma_{z z}$, which will not be needed. This restricted set of equations can be inverted to write the strains in terms of reduced compliances ${ }^{23} S_{i j}$,

$$
\begin{aligned}
& \varepsilon_{x x}=S_{11} \sigma_{x x}+S_{12} \sigma_{y y}, \\
& \varepsilon_{y y}=S_{12} \sigma_{x x}+S_{22} \sigma_{y y}, \\
& 2 \varepsilon_{x y}=S_{66} \sigma_{x y} .
\end{aligned}
$$

The coefficients have a capital letter as a reminder that they are not the usual compliances $s_{i j}$, which are found by inverting the complete set of stress-strain relations. The reduced compliances are given by

$$
\begin{aligned}
& S_{11}=\frac{c_{22}}{c_{11} c_{22}-c_{12}^{2}}, \\
& S_{22}=\frac{c_{11}}{c_{11} c_{22}-c_{12}^{2}}, \quad S_{12}=\frac{-c_{12}}{c_{11} c_{22}-c_{12}^{2}}, \quad S_{66}=\frac{1}{c_{66}} .
\end{aligned}
$$

The compatibility condition can now be written in terms of the stress as

$S_{11} \frac{\partial^{2} \sigma_{x x}}{\partial y^{2}}+S_{12} \frac{\partial^{2} \sigma_{y y}}{\partial y^{2}}-S_{66} \frac{\partial^{2} \sigma_{x y}}{\partial x \partial y}+S_{12} \frac{\partial^{2} \sigma_{x x}}{\partial x^{2}}+S_{22} \frac{\partial^{2} \sigma_{y y}}{\partial x^{2}}=0$,

which yields an equation for the Airy function,

$$
S_{11} \frac{\partial^{4} \chi}{\partial y^{4}}+\left(2 S_{12}+S_{66}\right) \frac{\partial^{4} \chi}{\partial x^{2} \partial y^{2}}+S_{22} \frac{\partial^{4} \chi}{\partial x^{4}}=0 .
$$

The coefficients obey $S_{11}=S_{22}$ with cubic aligned symmetry ${ }^{8,9,11}$ but the middle coefficient remains independent. In an isotropic medium the elastic constants fall out completely and Eq. (3.7) degenerates further to the biharmonic equation.

\section{B. Solution with complex variables}

Equation (3.7) for the Airy function is homogeneous and its general solution may therefore be written in the form

$$
\chi=f\left(\zeta_{1}\right)+g\left(\zeta_{2}\right)+\text { c.c. . }
$$

Here $f$ and $g$ are arbitrary complex functions and "c.c." means the complex conjugate, which is added to ensure that $\chi$ is real. The complex variables are defined by

$$
\zeta_{1}=x+i \beta_{1} y, \quad \zeta_{2}=x+i \beta_{2} y,
$$

where the constants $\beta_{1}$ and $\beta_{2}$ are roots of 


$$
S_{11} \beta^{4}-\left(2 S_{12}+S_{66}\right) \beta^{2}+S_{22}=0 .
$$

This is a quadratic equation in $\beta^{2}$ and therefore has two pairs of roots for $\beta$ with opposite signs. The values of $\beta_{1}$ and $\beta_{2}$ are selected to have positive real parts so that the region of interest, the upper half plane of $\zeta=x+i y$, is mapped to the upper half planes of $\zeta_{1}$ and $\zeta_{2}$. Also, the coefficients in Eq. (3.10) are real, in which case $\beta_{1}$ and $\beta_{2}$ are either both real or a pair of complex conjugates. It turns out that the roots themselves are not needed to find the elastic field at the surface; only their sum and product are required. The standard expressions for the sum and product of the roots of a quadratic equation provide the useful identities

$$
\begin{aligned}
& \beta_{1}^{2}+\beta_{2}^{2}=\frac{2 S_{12}+S_{66}}{S_{11}}=\frac{c_{11} c_{22}-c_{12}^{2}-2 c_{12} c_{66}}{c_{22} c_{66}}, \\
& \beta_{1}^{2} \beta_{2}^{2}=\frac{S_{22}}{S_{11}}=\frac{c_{11}}{c_{22}} .
\end{aligned}
$$

The stress is then given by the derivatives of the Airy function in Eq. (3.1),

$$
\begin{aligned}
& \sigma_{x x}=-\beta_{1}^{2} f^{\prime \prime}\left(\zeta_{1}\right)-\beta_{2}^{2} g^{\prime \prime}\left(\zeta_{2}\right)+\text { c.c. }, \\
& \sigma_{x y}=-i \beta_{1} f^{\prime \prime}\left(\zeta_{1}\right)-i \beta_{2} g^{\prime \prime}\left(\zeta_{2}\right)+\text { c.c. }, \\
& \sigma_{y y}=f^{\prime \prime}\left(\zeta_{1}\right)+g^{\prime \prime}\left(\zeta_{2}\right)+\text { c.c.. }
\end{aligned}
$$

The strain follows from the strain-stress relations in Eq. (3.4),

$$
\begin{aligned}
& \varepsilon_{x x}=\left(S_{12}-S_{11} \beta_{1}^{2}\right) f^{\prime \prime}\left(\zeta_{1}\right)+\left(S_{12}-S_{11} \beta_{2}^{2}\right) g^{\prime \prime}\left(\zeta_{2}\right)+\text { c.c. }, \\
& \varepsilon_{y y}=\left(S_{22}-S_{12} \beta_{1}^{2}\right) f^{\prime \prime}\left(\zeta_{1}\right)+\left(S_{22}-S_{12} \beta_{2}^{2}\right) g^{\prime \prime}\left(\zeta_{2}\right)+\text { c.c. },
\end{aligned}
$$

$$
2 \varepsilon_{x y}=-i S_{66}\left[\beta_{1} f^{\prime \prime}\left(\zeta_{1}\right)+\beta_{2} g^{\prime \prime}\left(\zeta_{2}\right)\right]+\text { c.c. . }
$$

Finally, the strain can be integrated to yield the displacement,

$$
\begin{aligned}
& u_{x}=\left(S_{12}-S_{11} \beta_{1}^{2}\right) f^{\prime}\left(\zeta_{1}\right)+\left(S_{12}-S_{11} \beta_{2}^{2}\right) g^{\prime}\left(\zeta_{2}\right)+\text { c.c. }, \\
& u_{y}=\frac{S_{22}-S_{12} \beta_{1}^{2}}{i \beta_{1}} f^{\prime}\left(\zeta_{1}\right)+\frac{S_{22}-S_{12} \beta_{2}^{2}}{i \beta_{2}} g^{\prime}\left(\zeta_{2}\right)+\text { c.c.. }
\end{aligned}
$$

The usual bodily shift and rotation can be added to the displacement.

\section{Boundary conditions}

It remains to find the potential in terms of the stress on the free surface. The expressions for the components of stress at the surface can be simplified by forming the linear combinations $^{22}$

$$
V(\zeta)=2\left[f^{\prime \prime}(\zeta)+g^{\prime \prime}(\zeta)\right], \quad W(\zeta)=2\left[\beta_{1} f^{\prime \prime}(\zeta)+\beta_{2} g^{\prime \prime}(\zeta)\right]
$$

Note that all functions depend on the same variable here and that all complex variables coincide on the surface, where $\zeta_{1}=\zeta_{2}=\zeta=x$. The boundary condition becomes

$$
\begin{aligned}
& \Re V(x+i \epsilon)=\sigma_{y y}(x, 0)=p(x), \\
& \mathfrak{I} W(x+i \epsilon)=\sigma_{x y}(x, 0)=q(x) .
\end{aligned}
$$

An infinitesimal imaginary part has been added to the arguments of $V$ and $W$ to keep them off the real axis. These functions can be continued analytically throughout the upper half plane, giving

$$
V(\zeta)=\frac{1}{\pi i} \int_{-\infty}^{\infty} \frac{p(t) d t}{t-\zeta}, \quad W(\zeta)=\frac{1}{\pi} \int_{-\infty}^{\infty} \frac{q(t) d t}{t-\zeta} .
$$

They have cuts on the real axis in regions where $p(x)$ and $q(x)$ are nonzero. The original functions are recovered as

$$
f^{\prime \prime}\left(\zeta_{1}\right)=\frac{W\left(\zeta_{1}\right)-\beta_{2} V\left(\zeta_{1}\right)}{2\left(\beta_{1}-\beta_{2}\right)}, \quad g^{\prime \prime}\left(\zeta_{2}\right)=-\frac{W\left(\zeta_{2}\right)-\beta_{1} V\left(\zeta_{2}\right)}{2\left(\beta_{1}-\beta_{2}\right)} .
$$

This completes the solution for the Airy function.

If the applied forces are purely normal to the surface, as for the strained slab, both $q$ and $W$ vanish. Then $f(\zeta)$ and $g(\zeta)$ have the same functional form, differing only in the prefactor. A similar simplification occurs for pure shear loading.

\section{Elastic field on surface}

The elastic field on the surface can now be found. Two components of stress, $\sigma_{y y}$ and $\sigma_{x y}$, are specified and the third component of interest is given by

$$
\begin{aligned}
\sigma_{x x}(x, 0) & =-\beta_{1}^{2} f^{\prime \prime}(x)-\beta_{2}^{2} g^{\prime \prime}(x)+\text { c.c. } \\
& =\beta_{1} \beta_{2} \Re V(x)-\left(\beta_{1}+\beta_{2}\right) \Re W(x) \\
& =\beta_{1} \beta_{2} p(x)-\left(\beta_{1}+\beta_{2}\right) \frac{1}{\pi} \mathcal{P} \int_{-\infty}^{\infty} \frac{q(t) d t}{t-x},
\end{aligned}
$$

where $\mathcal{P}$ denotes the principal part of the integral. For a purely normal applied stress this shows that

$$
\sigma_{x x}(x, 0)=\sqrt{\frac{c_{11}}{c_{22}}} \sigma_{y y}(x, 0),
$$

where Eq. (3.12) has been used to eliminate $\beta_{1} \beta_{2}$. This extends the result $\sigma_{x x}(x, 0)=\sigma_{y y}(x, 0)$ for isotropic media. ${ }^{14}$ The strains on the surface are

$$
\begin{aligned}
\varepsilon_{x x}(x, 0)= & \left(S_{11} \beta_{1} \beta_{2}+S_{12}\right) p(x) \\
& -S_{11}\left(\beta_{1}+\beta_{2}\right) \frac{1}{\pi} \mathcal{P} \int_{-\infty}^{\infty} \frac{q(t) d t}{t-x}, \\
\varepsilon_{y y}(x, 0)= & \left(S_{12} \beta_{1} \beta_{2}+S_{22}\right) p(x) \\
& -S_{12}\left(\beta_{1}+\beta_{2}\right) \frac{1}{\pi} \mathcal{P} \int_{-\infty}^{\infty} \frac{q(t) d t}{t-x}, \\
2 \varepsilon_{x y}(x, 0)= & S_{66} q(x) .
\end{aligned}
$$

For a purely normal applied stress, the strain is a local function of the stress and reduces to 


$$
\varepsilon_{x x}(x, 0)=\frac{p(x)}{\sqrt{c_{11} c_{22}}+c_{12}}, \quad \varepsilon_{y y}(x, 0)=\sqrt{\frac{c_{11}}{c_{22}}} \varepsilon_{x x}(x, 0) .
$$

These components are equal in an isotropic medium. ${ }^{14}$ The slope of the surface is

$$
\begin{aligned}
-\frac{\partial u_{y}(x, 0)}{\partial x}= & -\frac{S_{22}\left(\beta_{1}+\beta_{2}\right)}{\beta_{1} \beta_{2}} \Im V(x+i \epsilon) \\
& +\frac{S_{22}+S_{12} \beta_{1} \beta_{2}}{\beta_{1} \beta_{2}} \Im W(x+i \epsilon) \\
= & \frac{S_{22}\left(\beta_{1}+\beta_{2}\right)}{\beta_{1} \beta_{2}} \frac{1}{\pi} \mathcal{P} \int_{-\infty}^{\infty} \frac{p(t) d t}{t-x}+\frac{S_{22}+S_{12} \beta_{1} \beta_{2}}{\beta_{1} \beta_{2}} q(x) .
\end{aligned}
$$

A negative sign has been inserted because the outward displacement is $-u_{y}$ with the axes used. The slope is proportional to the locally applied shear stress and is a Hilbert transform of the normal stress. The transform can be inverted to deduce $p(x)$, and hence the composition of the slab, from the slope of the surface (paper I). Integration shows that the outward displacement due to a purely normal stress is

$$
-u_{y}(x, 0)=-\frac{S_{22}\left(\beta_{1}+\beta_{2}\right)}{\beta_{1} \beta_{2}} \frac{1}{\pi} \mathcal{P} \int_{-\infty}^{\infty} p(t) \ln |t-x| d t .
$$

\section{COMPLETE SOLUTION FOR RELAXATION OF THE SLAB}

For the relaxation of the slab we need the response to a purely normal applied stress $p(x)=P_{0}$ for $|x|<a$ and $p(x)$ $=0$ elsewhere, where $P_{0}$ is given by Eq. (2.4). It follows from the local nature of the strain in Eq. (3.23) that the cladding $(|x|>a)$ is unstrained at the surface. The surface of the slab has a uniform strain of

$$
\begin{aligned}
& \varepsilon_{x x}^{(\mathrm{rel})}=\frac{P_{0}}{\sqrt{c_{11} c_{22}}+c_{12}}=\frac{c_{11}\left(c_{22}+c_{23}\right)-c_{12}\left(c_{12}+c_{13}\right)}{c_{11}\left(\sqrt{c_{11} c_{22}}+c_{12}\right)} \varepsilon_{0}, \\
& \varepsilon_{y y}^{(\mathrm{rel})}=\sqrt{\frac{c_{11}}{c_{22}}} \varepsilon_{x x}^{(\mathrm{rel})} .
\end{aligned}
$$

The total strain of the slab at the surface is given by adding the strain deep within the slab, treated in Sec. II, to these results. This gives

$$
\begin{aligned}
& \frac{\varepsilon_{x x}^{\text {(tot) }}}{\varepsilon_{0}}=\frac{c_{11}\left(c_{22}+c_{23}\right)+\left(c_{12}+c_{13}\right) \sqrt{c_{11} c_{22}}}{c_{11}\left(\sqrt{c_{11} c_{22}}+c_{12}\right)}, \\
& \frac{\varepsilon_{y y}^{\text {(tot) }}}{\varepsilon_{0}}=-\frac{c_{12}\left(c_{12}+c_{13}\right)-c_{11} c_{23}+c_{12} \sqrt{c_{11} c_{22}}}{\sqrt{c_{11} c_{22}}\left(\sqrt{c_{11} c_{22}}+c_{12}\right)}, \\
& \frac{\varepsilon_{z z}^{\text {(tot) }}}{\varepsilon_{0}}=-1,
\end{aligned}
$$

$$
\frac{\varepsilon^{(\mathrm{tot})}}{\varepsilon_{0}}=\frac{\left(c_{22}-c_{12}\right)\left(c_{12}+c_{13}\right)-c_{11}\left(c_{22}-c_{23}\right)+\left(c_{22}+c_{23}-2 c_{12}\right) \sqrt{c_{11} c_{22}}}{\sqrt{c_{11} c_{22}}\left(\sqrt{c_{11} c_{22}}+c_{12}\right)} .
$$

A useful check on the total strains follows from the absence of "physical" traction on the free surface [as opposed to the fictitious traction $p(x)$ used to construct the solution]:

$$
0=\sigma_{y y}^{(\mathrm{tot})}=c_{12} \varepsilon_{x x}^{(\mathrm{tot})}+c_{22} \varepsilon_{y y}^{(\mathrm{tot})}+c_{23} \varepsilon_{z z}^{(\mathrm{tot})} .
$$

The outward relaxation is given by integrating Eq. (3.26) over the width of the slab, $|x|<a$. The result has the form of Eq. (1.1) with the constant given by

$$
\begin{aligned}
A_{0}= & \frac{S_{22}\left(\beta_{1}+\beta_{2}\right) P_{0}}{\beta_{1} \beta_{2}}=\frac{c_{11}\left(c_{22}+c_{23}\right)-c_{12}\left(c_{12}+c_{13}\right)}{\sqrt{c_{11} c_{22}}+c_{12}} \\
& \times\left[\frac{\sqrt{c_{11} c_{22}}+c_{12}+2 c_{66}}{c_{11} c_{66}\left(\sqrt{c_{11} c_{22}}-c_{12}\right)}\right]^{1 / 2} .
\end{aligned}
$$

The complex potentials are needed if the elastic field throughout the half space is required. There is no shear stress on the surface, $q(x)=0$, and therefore $W=0$. The other function $V$ is found by integrating Eq. (3.18) with $p(x)=P_{0}$ for $|x|<a$ and 0 otherwise, which yields

$$
V(\zeta)=\frac{P_{0}}{\pi i} \ln \frac{\zeta-a}{\zeta+a} .
$$

The functions $f^{\prime \prime}$ and $g^{\prime \prime}$ follow from Eq. (3.19) and are both proportional to $V$ because of the absence of shear traction. A useful check is to deduce the slope of the surface from Eq. (3.24), which gives

$$
-\frac{\partial u_{y}(x, 0)}{\partial x}=-\frac{S_{22}\left(\beta_{1}+\beta_{2}\right)}{\beta_{1} \beta_{2}} \Im V(x+i \epsilon)=\frac{A_{0}}{\pi} \ln \left|\frac{x-a}{x+a}\right| .
$$

This is consistent with the displacement in Eqs. (1.1) and (4.8).

\section{EFFECT OF SYMMETRY}

These general results are for orthotropic symmetry, where there are nine independent elastic coefficients. Different aspects of the elastic field depend on various subsets of coefficients: 
- $c_{11}, c_{22}$, and $c_{12}$ are needed to find the strains on the surface due to relaxation, given the initial stress $P_{0}$;

- $c_{66}$ is also needed to find the outward displacement of the surface and the elastic field throughout the sample due to relaxation;

- $\quad c_{13}$ and $c_{23}$ are also needed to find $P_{0}$ when the slab is matched to its cladding and to deduce $\sigma_{z z}^{(\mathrm{rel})}$ if it is needed;

- $c_{33}$ affects only the initial stress $\sigma_{z z}^{(\text {slab) }}$ and does not influence the relaxation; and

- $c_{44}$ and $c_{55}$ are not needed at all for this problem because the corresponding components of strain vanish.

Many practical systems exhibit higher symmetry, which introduces relations between these coefficients that may simplify the results.

\section{A. Tetragonal symmetry}

A material with a fourfold axis of rotation about $x$, the direction of growth, has only six independent elastic coefficients; they are related by $c_{12}=c_{13}, c_{22}=c_{33}$, and $c_{55}=c_{66}$. Only the first of these relations simplifies the results and the changes are not dramatic.

\section{B. Hexagonal symmetry and transverse isotropy}

Some nitride semiconductors exhibit hexagonal symmetry about the $x$ axis, which is the same as transverse isotropy for elasticity. It induces the further relation $c_{44}=\frac{1}{2}\left(c_{22}-c_{23}\right)$ and reduces the number of independent coefficients to five. Unfortunately it leads to no further simplification because $c_{44}$ does not affect the elastic field; $c_{66}$ is the relevant coefficient in these axes.

The slab is usually normal to hexagonal axis, which is labeled $x$ here but is conventionally taken as $z$. The elastic coefficients in the axes used here and in the conventional axes are related as follows:

$$
\begin{aligned}
& c_{11}=c_{33}^{(\text {hex })}, \\
& c_{22}=c_{33}=c_{11}^{(\text {hex })}, \\
& c_{12}=c_{13}=c_{13}^{(\text {hex })}, \\
& c_{23}=c_{12}^{(\text {hex })}, \\
& c_{44}=c_{66}^{(\text {hex })}=\frac{1}{2}\left[c_{11}^{(\text {hex })}-c_{12}^{(\text {hex })}\right], \\
& c_{55}=c_{66}=c_{44}^{(\text {hex })} .
\end{aligned}
$$

\section{Cubic rotated}

This applies to the usual cleaved $\{011\}$ surfaces of cubic semiconductors. The $x$ axis lies along the cubic axis [100] but the $y$ and $z$ axes are rotated by $45^{\circ}$ about $x$ from the cubic axes [010] and [001]. There are only three independent coefficients in a cubic material but six different values enter the stress-strain relations expressed in the $x y z$ axes because of the rotation. The elastic constants in these axes are given in terms of the cubic values by

$$
\begin{aligned}
& c_{11}=c_{11}^{(\mathrm{cub})}, \\
& c_{22}=c_{33}=\frac{1}{2}\left[c_{11}^{(\mathrm{cub})}+c_{12}^{(\mathrm{cub})}+2 c_{44}^{(\mathrm{cub})}\right], \\
& c_{12}=c_{13}=c_{12}^{(\mathrm{cub})}, \\
& c_{23}=\frac{1}{2}\left[c_{11}^{(\mathrm{cub})}+c_{12}^{(\mathrm{cub})}-2 c_{44}^{(\mathrm{cub})}\right], \\
& c_{44}=\frac{1}{2}\left[c_{11}^{(\mathrm{cub})}-c_{12}^{(\mathrm{cub})}\right], \\
& c_{55}=c_{66}=c_{44}^{(\mathrm{cub})} .
\end{aligned}
$$

The same pairs are equal as in tetragonal symmetry but with the further constraint that $c_{22}, c_{23}$, and $c_{44}$ are determined by the other coefficients.

\section{Cubic aligned}

In this case the $x y z$ and cubic axes coincide to give full cubic symmetry. There are only three independent elastic coefficients: $c_{11}=c_{22}=c_{33}, c_{12}=c_{13}=c_{23}$, and $c_{44}=c_{55}=c_{66}$. This leads to a considerable simplification of the results in Sec. IV, which become

$$
\begin{aligned}
& \frac{\varepsilon_{x x}^{(\mathrm{tot})}}{\varepsilon_{0}}=\frac{c_{11}+3 c_{12}}{c_{11}+c_{12}}, \\
& \frac{\varepsilon_{y y}^{(\mathrm{tot})}}{\varepsilon_{0}}=-\frac{2 c_{12}^{2}}{c_{11}\left(c_{11}+c_{12}\right)}, \\
& \frac{\varepsilon^{(\mathrm{tot})}}{\varepsilon_{0}}=\frac{2 c_{12}\left(c_{11}-c_{12}\right)}{c_{11}\left(c_{11}+c_{12}\right)}, \\
& A_{0}=\frac{c_{11}+2 c_{12}}{c_{11}+c_{12}}\left[\frac{\left(c_{11}-c_{12}\right)\left(c_{11}+c_{12}+2 c_{44}\right)}{c_{11} c_{44}}\right]^{1 / 2} .
\end{aligned}
$$

The components of strain on the surface due to relaxation become equal, $\varepsilon_{x x}^{(\mathrm{rel})}=\varepsilon_{y y}^{(\mathrm{rel})}$, as in an isotropic material. This follows from $c_{11}=c_{22}$ but is not obvious because the overall elastic field also depends on the independent coefficients $c_{12}$ and $c_{44}$.

\section{E. Isotropic symmetry}

Isotropic materials have only two independent coefficients and it is convenient to eliminate $c_{44}=\frac{1}{2}\left(c_{11}-c_{12}\right)$. The strains are unchanged from Sec. V D but the prefactor of the outward displacement can be reduced to

$$
A_{0}=2 \frac{c_{11}+2 c_{12}}{c_{11}+c_{12}} .
$$

The results can instead be written in terms of Poisson's ratio $\nu=c_{12} /\left(c_{11}+c_{12}\right)$, which gives

$$
\begin{aligned}
& \frac{\varepsilon_{x x}^{(\mathrm{tot})}}{\varepsilon_{0}}=1+2 \nu, \\
& \frac{\varepsilon_{y y}^{(\mathrm{tot})}}{\varepsilon_{0}}=-\frac{2 \nu^{2}}{1-\nu},
\end{aligned}
$$


TABLE I. Effect of symmetry on elastic constants, strain on surface, and prefactor for outward relaxation of surface; $\varepsilon_{z z}^{\text {(tot) }}=-\varepsilon_{0}$ in all cases. For comparison, the final row shows the strains deep within the slab.

\begin{tabular}{|c|c|c|c|c|c|c|c|c|}
\hline Symmetry & $\begin{array}{c}c_{11} \\
(\mathrm{GPa})\end{array}$ & $\begin{array}{c}c_{22} \\
(\mathrm{GPa})\end{array}$ & $\begin{array}{c}c_{12} \\
(\mathrm{GPa})\end{array}$ & $\begin{array}{c}c_{66} \\
(\mathrm{GPa})\end{array}$ & $\begin{array}{l}\varepsilon_{x x}^{(\mathrm{tot})} \\
\left(\varepsilon_{0}\right)\end{array}$ & $\begin{array}{l}\varepsilon_{y y}^{(\mathrm{tot})} \\
\left(\varepsilon_{0}\right)\end{array}$ & $\begin{array}{l}\varepsilon^{(\mathrm{tot})} \\
\left(\varepsilon_{0}\right)\end{array}$ & $\begin{array}{c}A_{0} \\
\text { (for } u_{y} \text { ) }\end{array}$ \\
\hline Isotropic & 114 & 114 & 53 & 30.5 & 1.634 & -0.295 & 0.339 & 2.634 \\
\hline Cubic aligned & 114 & 114 & 53 & 56.4 & 1.634 & -0.295 & 0.339 & 2.146 \\
\hline Cubic rotated & 114 & 140 & 53 & 56.4 & 1.586 & -0.407 & 0.179 & 1.864 \\
\hline Deep within slab & & & & & 0.930 & -1 & -1.070 & \\
\hline
\end{tabular}

$$
\begin{aligned}
& \frac{\varepsilon^{(\mathrm{tot})}}{\varepsilon_{0}}=\frac{2 \nu(1-2 \nu)}{1-\nu}, \\
& A_{0}=2(1+\nu) .
\end{aligned}
$$

These agree with I, where isotropic symmetry was assumed from the outset.

\section{PERIODIC VARIATION OF COMPOSITION}

No further calculation is needed for the strain on the surface if the composition has an arbitrary variation along $x$ because the fictitious stress $p(x)$ and resulting strains are local functions of the mismatch $\varepsilon_{0}(x)$. Unfortunately this does not apply to the outward relaxation, which requires either the full complex potential or the integral in Eq. (3.26).

\section{A. Sinusoidal variation}

This is useful to compare with previous calculations for cubic aligned symmetry, which used Fourier series. ${ }^{8,9,11}$ Take $\varepsilon_{0}(x)=\varepsilon_{0} \cos k x$, in which case the fictitious stress is $P(x)$ $=P_{0} \cos k x$ with $P_{0}$ again given by Eq. (2.4). It is useful to find the displacement from the complex potential rather than by integrating Eq. (3.25). The boundary condition (3.17) gives $\mathfrak{R} V(x+i \epsilon)=P_{0} \cos k x$. This is like an electrostatic problem where the potential is specified on the boundary. In the same way, it must be extended analytically into the complex plane such that the field decays as $y \rightarrow \infty$. Thus $V(\zeta)$ $=P_{0} \exp (i k \zeta)$. The slope of the surface is found using Eq. (3.24),

$$
-\frac{\partial u_{y}(x, 0)}{\partial x}=-\frac{S_{22}\left(\beta_{1}+\beta_{2}\right)}{\beta_{1} \beta_{2}} \Im V(x)=-A_{0} \sin k x,
$$

where $A_{0}$ is given by Eq. (4.8). Trivial integration yields $-u_{y}(x, 0)=A_{0} \varepsilon_{0} \cos k x / k$, which is equivalent to Eq. (3.26). This can be used with a Fourier expansion to find the displacement due to any form of $\varepsilon_{0}(x)$.

\section{B. Superlattice}

Instead of a single mismatched slab, suppose that there is a regular superlattice with slabs of thickness $2 a$ and mismatch $\varepsilon_{0}$ alternating with matched spacers of thickness $2 b$. Neglect the average mismatch between the superlattice and substrate and concentrate on the periodic component; the average mismatch of a finite superlattice can be treated as a simple slab and superposed. The complex potential of the periodic component is

$$
V(\zeta)=\frac{P_{0}}{\pi i} \log \frac{\sin \frac{1}{2} \pi(\zeta-a) /(a+b)}{\sin \frac{1}{2} \pi(\zeta+a) /(a+b)} .
$$

The slope of the surface follows immediately from Eq. (3.24),

$$
-\frac{\partial u_{y}(x, 0)}{\partial x}=\frac{A_{0}}{\pi} \log \left|\frac{\sin \frac{1}{2} \pi(x-a) /(a+b)}{\sin \frac{1}{2} \pi(x+a) /(a+b)}\right|,
$$

where $P_{0}$ and $A_{0}$ have their usual meanings. Unfortunately this cannot be integrated to give the displacement in terms of elementary functions, but can be expressed using either Lobachevsky's function (Sec. 8.26 of Ref. 24) or Clausen's integral $f(\theta)$ (Sec. 27.8 of Ref. 25). The peak-to-peak displacement is convenient to measure ${ }^{3}$ and is found to be

$$
u_{y}^{\mathrm{pk}-\mathrm{pk}}=\frac{2(a+b) A_{0}}{\pi^{2}}\left[f\left(\frac{\pi a}{a+b}\right)+f\left(\frac{\pi b}{a+b}\right)\right] .
$$

This has the form $f(\theta)+f(\pi-\theta)$, for which the following series $^{25}$ is helpful:

$$
f(\theta)+f(\pi-\theta)=\sum_{n \text { odd }} \frac{\sin n \theta}{n^{2}} .
$$

Fourier analysis leads to the same result. ${ }^{8,9,11}$ For a superlattice with slabs and spacers of equal thickness, $f\left(\frac{1}{2} \pi\right)$ $=G \approx 0.916$, where $G$ is Catalan's constant. ${ }^{25}$

\section{RESULTS}

The sample studied in I contained two quantum wells of $\mathrm{In}_{x} \mathrm{Ga}_{1-x}$ As surrounded by GaAs. The wider well had width $2 a=6.5 \mathrm{~nm}$ and composition $x=0.14$, determined by crosssectional scanning tunneling microscopy and $\mathrm{x}$-ray diffraction. The well is more stressed on average than its surroundings and the elastic constants of the well will therefore be used in the theory. Linear interpolation between the values for GaAs and InAs (Ref. 26) gives the values listed in Table I. The natural lattice constants are $a_{\text {cladding }}=0.565 \mathrm{~nm}$ and $a_{\text {slab }}=0.571 \mathrm{~nm}$, giving a built-in strain $\varepsilon_{0}=0.0108$ according to Eq. (2.2).

We shall compare the three cases of isotropic, cubic aligned, and cubic rotated materials to highlight the effect of symmetry on the relaxation. The results are summarized in Table I and will be compared with the numerical results and experiment in Sec. VIII. First, a value must be chosen for Poisson's ratio $\nu$ in the isotropic approximation. During the fictitious assembly of the sample, stress arises from the compression of the slab to match its cladding on the (100) plane. We therefore use the definition $\nu=c_{12}^{\text {(cub) }} /\left[c_{11}^{(\text {(cub) }}+c_{12}^{\text {(cub) }}\right]$ 
$=0.317$ to describe this process correctly. This is equivalent to setting $c_{11}^{\text {(iso) }}=c_{11}^{\text {(cub) }}, \quad c_{12}^{\text {(iso) }}=c_{12}^{\text {(cub) }}$, and $c_{44}^{\text {(iso) }}=1 / 2\left[c_{11}^{\text {(cub) }}\right.$ $\left.-c_{12}^{\text {(cub) }}\right]=30.5 \mathrm{GPa}$. The shear stiffness is much smaller than its true value of $c_{44}^{\text {(cub) }}=56.4 \mathrm{GPa}$. With this choice the strains and fictitious stress $P_{0}$ in the slab, given in Eqs. (2.3) and (2.4), are identical for all the three cases. Any differences result from the relaxation in response to $P_{0}$.

Within the isotropic approximation, the results in Sec. $\mathrm{V} E$ show that the strain along the direction of growth is given by $\varepsilon_{x x}^{\text {(tot) }} / \varepsilon_{0}=(1+2 \nu)=1.634$ and the prefactor for outward relaxation in Eq. $(1.1)$ is $A_{0}=2(1+\nu)=2.634$. These results agree with those in I except for small numerical differences because we used the elastic constants for the GaAs cladding previously instead of the InGaAs well.

We can now examine the changes induced by cubic symmetry, first considering the cubic aligned case where the crystal is cleaved on a $\{001\}$ plane. This does not apply to the common semiconductors but is a useful intermediate step and can be compared with previous calculations. ${ }^{8,9}$ The strain along the direction of growth is given by Eq. (5.3) and is unchanged from the isotropic value because of the way in which $\nu$ has been defined. Equation (5.6) for the prefactor for outward relaxation is different, however, and gives the smaller value $A_{0}=2.146$. This is reduced by about $20 \%$ from the isotropic case because of the greater shear stiffness $c_{44}^{\text {(cub) }}$ in cubic material, which has no effect at the surface but reduces relaxation throughout the bulk.

Finally, the cubic rotated case accounts for the $\{011\}$ orientation of the cleavage plane. Expressions for the elastic constants in these axes were given in Eq. (5.2) and the numerical values are listed in Table I. The strain along the direction of growth now follows from Eq. (4.3) as $\varepsilon_{x x}^{\text {(tot) } / \varepsilon_{0}}$ $=1.586$, which is slightly smaller than the value for isotropy. The prefactor for outward relaxation becomes $A_{0}=1.864$ from Eq. (4.8), a further reduction of about $10 \%$ from the cubic aligned case. Table I shows that the rotation by $45^{\circ}$ has increased the stiffness $c_{22}$, which is no longer equal to $c_{11}$, and this has reduced all aspects of relaxation.

To gain further insight into the effect of symmetry, the elastic field throughout the half space was calculated. The mathematical form of the fields changes with symmetry, unlike the expressions for the surface alone, and depends on the nature of the roots $\beta_{1}$ and $\beta_{2}$ of the quadratic equation (3.10). Here the roots are complex. The stress was found from the complex potential in Eq. (4.9) by using Eqs. (3.19) and (3.13), and the strain follows from Eq. (3.14).

Components of stress due to relaxation are shown for the three cases as a function of depth in Fig. 2. The normal stresses $\sigma_{x x}$ and $\sigma_{y y}$ are plotted for the midplane of the well, $x=0$, but the shear stress $\sigma_{x y}$ vanishes here by symmetry and is therefore plotted at the edge of the well, $x=a$. No shear traction is applied to the surface so the shear stress should vanish at $y=0$, but it is nonzero in Fig. 2(c) because of a singularity at the point $(a, 0)$. The components show different rates of decay with depth: for large depth $\sigma_{x x} \sim y^{-3}, \sigma_{x y}$ $\sim y^{-2}$, and $\sigma_{y y} \sim y^{-1}$.

The elastic constants have no influence at all on the stress in an isotropic two-dimensional system because Eq. (3.7) for the Airy function reduces to the biharmonic equa-

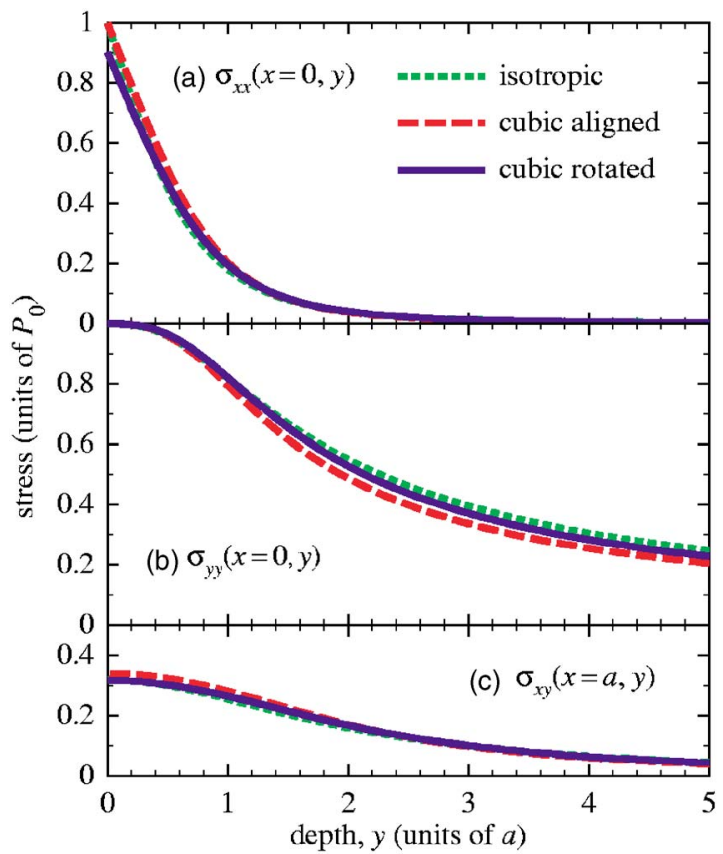

FIG. 2. (Color online) Components of stress due to relaxation as a function of depth $y$ : (a) $\sigma_{x x}(0, y)$ and (b) $\sigma_{y y}(0, y)$ in midplane of well, with (c) $\sigma_{x y}(a, y)$ at edge of well. The results are shown for the isotropic, cubic aligned, and cubic rotated cases. Stress is measured in units of the fictitious traction $P_{0}$ and distance in units of the half width $a$.

tion. This result is exact only in the isotropic limit but Fig. 2 shows that the effect of anisotropy is small in our system, no more than $10 \%$ of the maximum values of stress. The largest change is in $\sigma_{x x}(0,0)$, which is reduced in the cubic rotated case according to Eq. (3.21).

Symmetry has a larger effect on the strain, plotted in Fig. 3. Isotropic material shows the largest values of $\varepsilon_{x x}$ and $\varepsilon_{y y}$, which are reduced slightly in the cubic aligned orientation. This is consistent with the observation in Ref. 9 that strain changes by only about $6 \%$ between isotropic and cubic aligned symmetries. There is a larger difference between the two orientations of cubic material, particularly near the surface. This is consistent with the different magnitude of bulge at the surface, because the displacement $u_{y}$ is the integral of $\varepsilon_{y y}$. The behavior of the shear strain is different: the curves for the two orientations of cubic material are very close, but that for isotropic material is far away. This arises from the different values of the shear stiffness $c_{66}$, which is $56.4 \mathrm{GPa}$ for both orientations of cubic material but only $30.5 \mathrm{GPa}$ in the isotropic material, given our choice of $\nu$.

These results show that the strain is more sensitive to symmetry than the stress. A simple approximation is to calculate the stress for an isotropic material, which is easier, but to deduce the strain using the appropriate cubic elastic moduli, including the effect of symmetry and orientation. Results are shown as the thin lines in Fig. 3. The solid, thin lines for the cubic rotated case lie close to the thick lines for the exact solution for all three components almost everywhere; the only significant discrepancy is in $\varepsilon_{x x}(0,0)$, which follows from the reduction in $\sigma_{x x}(0,0)$ for cubic rotated symmetry. Table I shows that the elastic constants for normal stress and strain are the same in the isotropic and cubic 


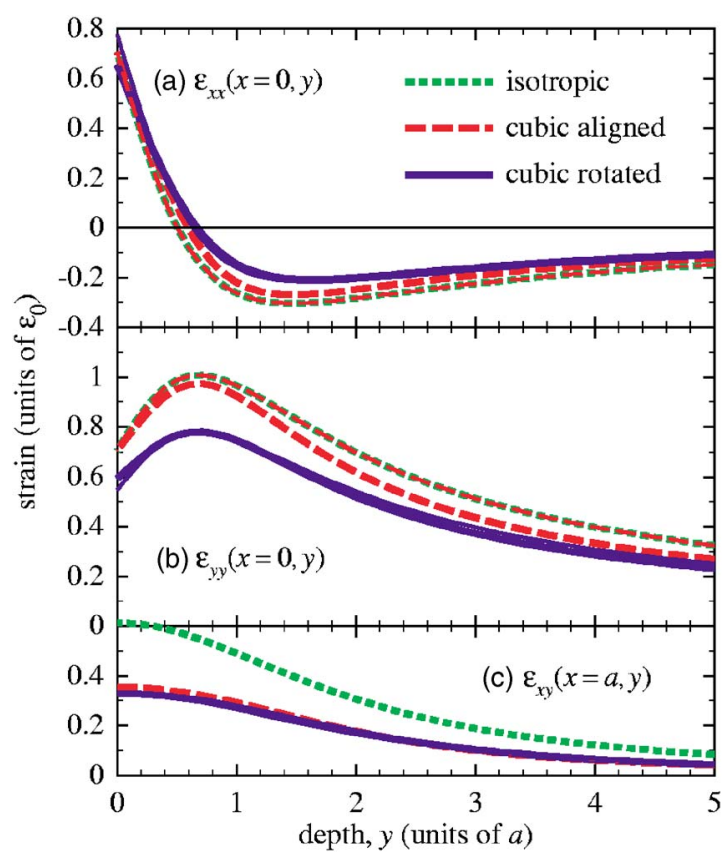

FIG. 3. (Color online) Components of strain due to relaxation as a function of depth $y$ : (a) $\varepsilon_{x x}(0, y)$ and (b) $\varepsilon_{y y}(0, y)$ in midplane of well, with (c) $\varepsilon_{x y}(a, y)$ at edge of well. The results are shown for the isotropic, cubic aligned, and cubic rotated cases. Thin lines show strain calculated from the stress for isotropic material using the elastic constants for cubic material in the two orientations. Strain is measured in units of the mismatch $\varepsilon_{0}$ and distance in units of the half width $a$.

aligned cases. Therefore the approximate solution for the cubic aligned case (long, thin dashes) is the same as the isotropic solution (short, thick dashes) for $\varepsilon_{x x}$ and $\varepsilon_{y y}$. This is not true for $\varepsilon_{x y}$, however. In this case the effective value of $c_{66}$ is the same for both orientations of cubic material because the shear stiffness does not change on rotation. The approximate solutions for cubic aligned and cubic rotated therefore coincide and are indistinguishable from the exact solution for cubic rotated symmetry on this scale.

\section{DISCUSSION}

We have calculated the influence of elastic symmetry on the relaxation of a strained layer, such as a mismatched quantum well, at a surface cleaved perpendicular to the layer. General results have been given for orthotropic symmetry, which includes the important cases of cubic crystals cleaved on $\{011\}$ or $\{001\}$ surfaces; orthorhombic, tetragonal, and hexagonal crystals; and the limit of isotropic material. The functional form of the elastic field depends on symmetry in the bulk but not at the surface, where only the magnitudes change. In particular, the lattice constant observed at the surface remains a local function of composition, even if this has an arbitrary variation along the direction of growth. Alternatively, the composition may be deduced from a Hilbert transform of the slope of the surface as shown in I.

Our previous calculation for isotropic material in I predicted a relaxation that was only about $80 \%$ of the observed value. This applied to both the lattice constants along the direction of growth on the surface and to the outward relaxation. Cubic symmetry in GaAs influences these quantities rather differently.



FIG. 4. (Color online) Numerical calculations of outward relaxation of surface using ABAQUS to show the effect of cubic symmetry, orientation, and of assuming that the elastic constants in the slab and cladding are identical. The experimental result from I is also shown. Curves have been shifted vertically to coincide near the edge of the plot because the displacement contains an arbitrary constant.

- There is no effect on the lattice constant at all if the surface is a $\{001\}$ plane and only a small change (about $3 \%$ ) for a $\{011\}$ plane, which has a greater stiffness $c_{22}$ normal to the surface.

- In contrast, the outward relaxation is reduced by about $20 \%$ by cubic symmetry for a $\{001\}$ plane because the shear stiffness is much higher than in the isotropic model. Rotation to a $\{011\}$ plane reduces the relaxation by a further $10 \%$.

Unfortunately these results show that the inclusion of cubic symmetry increases the discrepancy between the calculation and experiment. We advanced several possible reasons for the difference previously. One was the assumption of isotropic symmetry, which we have now addressed, and we shall briefly review some other possibilities.

We have performed numerical computations to test some of the assumptions of the analytical work. Figure 4 shows the outward relaxation of the surface calculated using the commercial finite-element package ABAQUS, assuming a concentration of indium $x=0.171$ in the wider well. This value was chosen to bring the calculations for isotropic elasticity into agreement with the experiment but is larger than the value of 0.14 deduced in I from counting atoms on the surface. All calculations were for the same region so that boundary effects should be similar; these are difficult to eliminate because of the slow decay of the elastic field, described in Sec. VII. Different elastic constants were used for the well and cladding in most cases. The first three curves show that the relaxation is smaller for cubic aligned material than for isotropic, and that cubic rotated is smaller still. This is consistent with the analytic results in Table I. The fourth (solid thin) curve was computed using the elastic constants for the slab everywhere, as assumed in the analytic calculation. The slab is a little softer than the cladding so this approximation gives a slightly larger relaxation than the solid thick curve, where different elastic constants were used for the well and cladding. The change is negligible compared with the difference between the calculated curves and the experimental results (dots), and therefore cannot explain the discrepancy.

Relaxation may be influenced by energies associated with the free surface and interfaces, which also have intrini- 
sic stress. It has been $\operatorname{argued}^{27}$ that these have a significant effect on InAs quantum dots in GaAs, because the intrinsic stress for a surface of InAs (Ref. 28) is around $0.8 \mathrm{~N} \mathrm{~m}^{-1}$. For comparison, the fictitious applied force is $2 P_{0} a$ $\approx 8 \mathrm{~N} \mathrm{~m}^{-1}$. Thus the interfacial stress is smaller but perhaps noticeable. However, relaxation is likely to be affected by the difference between GaAs and InGaAs. The surface stress for GaAs (Ref. 29) is roughly $1.0 \mathrm{~N} \mathrm{~m}^{-1}$ so the difference between GaAs and $\mathrm{In}_{0.14} \mathrm{Ga}_{0.86}$ As should only be around $0.03 \mathrm{~N} \mathrm{~m}^{-1}$, which is negligible.

The most likely explanation of the discrepancy between theory and experiment is that the elastic response is nonlinear. A calculation for InAs quantum dots in GaAs (Ref. 30) found that nonlinearity reduced the compression of the dot by $16 \%$ of its value within linear elasticity. The mismatch is smaller in our system but it is possible that the nature of the system enhances nonlinearity: the slab is first compressed to match it to the cladding, which increases its stiffness and raises $P_{0}$ above its value for linear elasticity; relaxation then dilates the slab near the surface, reducing its stiffness and increasing the distortion further. Unfortunately the highly inhomogeneous nature of the strain renders a numerical calculation essential.

\section{ACKNOWLEDGMENTS}

One of the authors (J.H.D.) would like to thank N. W. Ashcroft, S. L. Phoenix, J. P. Sethna, and H. Ustunel for discussions and the Laboratory of Applied and Solid State Physics at Cornell University for their hospitality. He is also grateful for helpful correspondence with D. A. Faux.

${ }^{1}$ P. G. Piva et al., Appl. Phys. Lett. 72, 1599 (1998).

${ }^{2}$ B. Legrand, B. Grandidier, J. P. Nuys, D. Stiévenard, J. M. Gérard, and V. Thierry-Mieg, Appl. Phys. Lett. 73, 96 (1998).
${ }^{3}$ H. Chen, R. M. Feenstra, R. S. Goldman, C. Silfvenius, and G. Landgren, Appl. Phys. Lett. 72, 1727 (1998).

${ }^{4}$ H. Chen, R. M. Feenstra, P. G. Piva, R. D. Goldberg, I. V. Mitchell, G. C. Aers, P. J. Poole, and S. Charbonneau, Appl. Phys. Lett. 75, 79 (1999). ${ }^{5}$ H. Chen et al., J. Appl. Phys. 89, 4815 (2001).

${ }^{6}$ J. H. Davies, D. M. Bruls, J. W. A. M. Vugs, and P. M. Koenraad, J. Appl. Phys. 91, 4171 (2002). Part I.

${ }^{7}$ M. M. J. Treacy, J. M. Gibson, and A. Howie, Philos. Mag. A 51, 389 (1985)

${ }^{8}$ M. M. J. Treacy and J. M. Gibson, J. Vac. Sci. Technol. B 4, 1458 (1986). ${ }^{9}$ D. A. Faux and J. Haigh, J. Phys.: Condens. Matter 2, 10289 (1990).

${ }^{10}$ D. A. Faux, J. Appl. Phys. 75, 186 (1994).

${ }^{11}$ L. De Caro, L. Tapfer, and A. Giuffrida, Phys. Rev. B 54, 10575 (1996).

${ }^{12}$ S. P. Timoshenko and J. N. Goodier, Theory of Elasticity, 3rd ed. (McGraw-Hill, New York, 1970).

${ }^{13}$ B. A. Boley and J. H. Weiner, Theory of Thermal Stresses (Dover, Mineola, NY, 1997).

${ }^{14}$ K. L. Johnson, Contact Mechanics (Cambridge University Press, Cambridge, UK, 1985).

${ }^{15}$ M. Tadic, F. M. Peeters, and K. L. Janssens, Phys. Rev. B 65, 165333 (2002).

${ }^{16}$ E. Pan and B. Yang, J. Appl. Phys. 90, 6190 (2001).

${ }^{17}$ E. Pan, J. Appl. Phys. 91, 3785 (2002).

${ }^{18}$ C. Q. Ru, Proc. R. Soc. London, Ser. A 456, 1051 (2000).

${ }^{19}$ E. Pan, J. Appl. Phys. 91, 6379 (2002).

${ }^{20}$ E. Pan and B. Yang, J. Appl. Phys. 93, 2435 (2003).

${ }^{21}$ D. A. Faux and J. Gill, J. Appl. Phys. 75, 4963 (1994)

${ }^{22}$ A. E. Green and W. Zerna, Theoretical Elasticity, 2nd ed. (Oxford University Press, Oxford, UK, 1968).

${ }^{23}$ T. C. T. Ting, Anisotropic Elasticity: Theory and Applications (Oxford University Press, New York, 1996).

${ }^{24}$ I. S. Gradshteyn and I. M. Ryzhik, Table of Integrals, Series, and Products, 6th ed. (Academic, San Diego, 2000).

${ }^{25}$ Handbook of Mathematical Functions, edited by M. Abramowitz and I. A. Stegun (National Bureau of Standards, Washington, 1970).

${ }^{26}$ Semiconductors - Basic Data, edited by O. Madelung (Springer, Berlin, 1996).

${ }^{27}$ P. Sharma and S. Ganti, Phys. Status Solidi B 234, R10 (2002).

${ }^{28}$ N. Moll, M. Scheffler, and E. Pehlke, Phys. Rev. B 58, 4566 (1998).

${ }^{29}$ N. Moll, A. Kley, E. Pehlke, and M. Scheffler, Phys. Rev. B 54, 8844 (1996).

${ }^{30}$ S. W. Ellaway and D. A. Faux, J. Appl. Phys. 92, 3027 (2002). 